Pacific Journal of Mathematics

A PROBABILISTIC METHOD FOR THE RATE OF
CONVERGENCE TO THE DIRICHLET PROBLEM

DAVID F. ERASE 


\title{
A PROBABILISTIC METHOD FOR THE RATE OF CON- VERGENCE TO THE DIRICHLET PROBLEM
}

\author{
DAVId F. Fraser
}

The expectation $E^{p}(\Phi)$ approximates the solution $u(z)=$ $E^{W}(\Phi)$ of the Dirichlet problem for a plane domain $D$ with boundary conditions $\phi$ on the boundary $\gamma$ of $D$, where $W$ is Wiener measure, $P$ is the measure generated by a random walk which approximates Brownian motion beginning at $z$, and $\Phi$ is the functional on paths which equals the value of $\phi$ at the point where the path first meets $\gamma$. This paper develops a specific rate of convergence. If $\gamma$ is $C^{2}$, and $P^{n}$ is generated by random walks beginning at $z$, with independent increments in the coordinate directions at intervals $1 / n$, with mean zero, variance $1 / \sqrt{n}$, and absolute third moment bounded by $M$, then $\mid E^{P^{n}}(\Phi)-$ $E^{W}(\Phi) \mid \leqq(C M V \mid \rho(z, \gamma)) n^{-1 / 16}(\log n)^{9 / 8}$, where $V$ is the total variation of $\phi$ on $\gamma, \rho(z, \gamma)$ is the distance from $z$ to $\gamma$, and $C$ is a constant depending only on $\gamma$.

Assume $D$ is a Jordan region. If $z_{t}=x_{t}+i y_{t}$ is Brownian motion in $R^{2}$ beginning at $z_{0}$, (cf. e.g., [5, p. 262]), and $\tau=$ $\inf \left\{t: z_{t} \in \gamma\right\}$ is the first time $z$ hits the boundary $\gamma$ of $D$, then $\Phi$ is the functional given by $\Phi\left(z_{\bullet}\right)=\phi\left(z_{\tau}\right)$. Let $E^{W}\left(\Phi\left(z_{\bullet}\right)\right)=\int \Phi\left(z_{\bullet}\right) d W$ be the expectation of $\Phi$ with respect to Wiener measure $W$ on $C([0, \infty)$, C). (See [8, pp. 218-19] for a definition of Brownian motion on the interval $[0,1]$ and the corresponding Wiener measure.)

Let $g_{1}^{1}, g_{1}^{2}, g_{2}^{1}, g_{2}^{2}, \cdots, g_{k}^{1}, g_{k}^{2}, \cdots$ be a sequence of indendent random variables with mean zero, variance 1 , and absolute third moment bounded uniformly by $M<\infty$, and let

$$
\xi_{i}^{\alpha}=g_{i}^{\alpha} / \sqrt{n}, \zeta_{0}=z_{0}, \zeta_{k}=z_{0}+\sum_{i=1}^{k}\left(\xi_{i}^{1}+\sqrt{-1 \xi_{i}^{2}}\right), t_{k}=k / n .
$$

Let $\xi(t)$ be the continuous random broken line which has vertices $\left(t_{k}, \zeta_{k}\right)$ and is linear between vertices. Let $P^{n}$ be the measure on $C\left([0, \infty), C^{\prime}\right)$ generated by this line, i.e., $P^{n}(S)=P(\xi(t) \in S)$.

Now by the Central Limit Theorem $P^{n}\left(\xi^{\alpha}(t) \leqq \lambda\right) \rightarrow W\left(z_{t}^{\alpha} \leqq \lambda\right)$, $\alpha=1,2$, where $\xi^{\alpha}(t), z_{t}^{\alpha}$ are the real and imaginary parts of $\xi(t), z_{t}$ respectively, (cf. e.g., [1, pp. 186-7]). More exactly one has the Barry-Esseen Theorem [3, p. 521]: For nt an integer

$$
\sup _{\lambda}\left|P\left(\xi^{\alpha}(t) \leqq \lambda\right)-N(\lambda / \sqrt{t})\right| \leqq \frac{33}{4} M / \sqrt{n t}
$$

where $N(x)$ is the normal distribution. A useful generalization of the 
Central Limit Theorem is that convergence also takes place for the expectation of any functional on $C[0,1)$ which is continuous with respect to uniform convergence on $[0,1]$ and satisfies mild growth conditions, e.g. $\Phi(x)=\int_{0}^{1} x(t)^{2} d t, \sup _{0 \leqq t \leqq 1} x_{t}$, etc. Rates of convergence have been calculated for some specific one-dimensional functionals $\Phi$, (e.g., [10], [11]). For an arbitrary functional $\Phi$ satisfying a uniform Hölder condition one can get rates of convergence using Levy distance in $C[0, t]$ ([9], see also $\S 2$ of this paper). Explicit rates of convergence are of interest for various practical problems and computer applications.

Although $\Phi(z)=\phi\left(z_{\tau}\right)$ is not continuous with respect to uniform convergence, it is continuous a.s. with respect to Wiener measure, so convergence takes place. In this paper we obtain a rate of convergence.

THEOREM. There exists a universal constant $C^{*}=C^{*}(\gamma)$ such that

$$
\left|E^{P^{n}}(\Phi)-E^{W}(\Phi)\right| \leqq \frac{C^{*} V(\phi) M}{\rho\left(z_{0}, \gamma\right)} n^{-1 / 16}(\log n)^{9 / 8}
$$

where $V(\phi)$ is the total variation of $\phi$ on $\gamma, M$ is the bound on absolute third moments defined above, $z_{0}$ is the initial point of the paths $z_{\text {., }}$ and $\rho\left(z_{0}, \gamma\right)=\inf _{s}\left|z_{0}-\gamma(s)\right|$ is the distance from $z_{0}$ to $\gamma$.

2. Levy distance. We define measures $P_{t}^{n}, W_{t}$ on $C([0, t], \mathscr{C})$ by

$$
P_{t}^{n}(S)=P^{n}\left(\pi^{-1} S\right), W_{t}(S)=W\left(\pi^{-1} S\right),
$$

where $\pi: C([0, \infty), \mathscr{C}) \rightarrow C([0, t], \mathscr{C})$ is the projection $\pi(f)=\left.f\right|_{[0, t]}$. The Levy distance $L$ between the measures $P_{t}^{n}$ and $W_{t}$ is given by

$$
L\left(P_{t}^{n}, W_{t}\right)=\max \left(\varepsilon_{1}, \varepsilon_{2}\right),
$$

where

$$
\begin{aligned}
& \varepsilon_{1}=\inf \left\{\varepsilon: P_{t}^{n}(S) \leqq W_{t}\left(S^{s, t}\right)+\varepsilon \text { for all closed sets } S\right\} \\
& \varepsilon_{2}=\inf \left\{\varepsilon: W_{t}(S) \leqq P_{t}^{n}\left(S^{s, t}\right)+\varepsilon \text { for all closed sets } S\right\}
\end{aligned}
$$

and

$$
S^{s, t}=\left\{y: \exists x \in S \ni \sup _{0 \leqq s \leqq t}|y(s)-x(s)|<\varepsilon\right\}
$$

is an $\varepsilon$-neighborhood of $S$ with respect to the sup-norm on $[0, t]$.

The following proposition is a direct generalization of a result of Prokhorov ([9]) to two dimensions as is its proof.

Proposition 1. There exists an absolute constant $C$ such that

$$
L\left(P_{1}^{n}, W_{1}\right) \leqq C M^{1 / 4} n^{-1 / 8}(\log n)^{15 / 8} .
$$




\section{COROLLARY.}

If $t=k / n, k$ an integer, then $L\left(P_{t}^{n}, W_{t}\right) \leqq C \sqrt{t} k^{-1 / 8}(\log k)^{15 / 8}$ for some constant $C$.

3. Boundedness of harmonic density. Fix a point $\gamma_{0}$ on $\gamma$, and a direction along $\gamma$, parametrize $\gamma$ by arclength in the chosen direction from $\gamma_{0}$. Let $l$ denote the length of $\gamma$, and take the argument $s$ of $\gamma=\gamma(s)$ modulo $l$.

Since $\gamma$ is $C^{2}$, there exists $R>0$ such that any circle of radius $R$ will meet $\gamma$ in at most two points. It follows that for any two points $\gamma(a)$ and $\gamma(a+\delta)$ on $\gamma$ where $0<\delta<R$ that $\gamma([a, a+\delta])$ will lie in the intersection of the closed disks bounded by the two circles of radius $R$ through $\gamma(a)$ and $\gamma(a+\delta)$. The case we have to eliminate is where $\gamma$ is tangent to one of the circles at $\gamma(a)$ and $\gamma(a+$ $\delta)$, but does not cross the circle, i.e., there are neighborhoods in $\gamma$ of $\gamma(a)$ and $\gamma(a+\delta)$ which do not meet the closed disk bounded by the circle except at $\gamma(a)$ or $\gamma(a+\delta)$. But in this case we observe that a small rotation of the circle about one of the points $\gamma(\alpha)$ or $\gamma(\alpha+\delta)$ will result in three points of intersection, contradicting our assumption about $\gamma$. Furthermore, it follows from the Jordan curve theorem that the center of one of the two circles will be in $D$, the other center will be outside $D$.

We are now ready to prove the following result.

Proposition 2.

$$
W\left(z_{\tau} \in \gamma([a, a+\delta])\right) \leqq B \delta / \rho\left(z_{0}, \gamma\right)
$$

where $B$ is an absolute constant depending only on $\gamma$.

Proof. We may assume $\delta<R$ and also $\delta$ sufficiently small that

$$
2\left(R-\left(R^{2}-\delta^{2} / 4\right)^{1 / 2}\right)<\rho\left(z_{0}, \gamma\right) / 2,
$$

since by addition if the proposition holds for small $\delta$, it holds for $\delta$ in general.

Let $C$ be the circle of radius $R$ through $\gamma(a)$ and $\gamma(a+\delta)$, with center not in $D$. Then

$$
P_{z_{0}}\left(z_{\tau} \in \gamma([a, a+\delta])\right) \leqq P_{z_{0}}\left(z_{\tau(C)} \in \delta^{*}\right)
$$

where $\tau(C)=\inf \left\{t: z_{t} \in C\right\}$, and $\delta^{*}=D \cap C$. Now invert the plane with respect to the circle $C$, sending $z_{0}$ into $I\left(z_{0}\right)$. Now $I\left(z_{t}\right)$ is Brownian motion with a time change. (P. Lévy [7, p. 254], see also [5, pp. 279-80] for another proof of this.) However, where $I\left(z_{t}\right)$ first hits $C$ is independent of any time change; "Les proprietés intrinsèques de 
la courbe $C$ sont invariantes par une representation conforme." Now

$$
z_{\tau(C)} \in C \Longrightarrow I\left(z_{\tau(C)}\right)=z_{\tau(C)}, I\left(\delta^{*}\right)=\delta^{*},
$$

so $P_{z_{0}}\left(z_{\tau(C)} \in \delta^{*}\right)=P_{z_{0}}\left((I(z))_{\tau^{\prime}(C)} \in \delta^{*}\right)$ where $\tau^{\prime}(C)=\inf \left\{s:(I(z))_{s} \in C\right\}$.

But the harmonic density on a circle is given by the Poisson kernel (cf. e.g., [4, p. $361 \mathrm{ff.}$ ); it is bounded,

$$
P_{z_{0}}\left((I(z))_{z^{\prime}(C)} \in \delta^{*}\right) \leqq \frac{2}{2 \pi}\left|\delta^{*}\right| / \rho\left(I\left(z_{0}\right), C\right)
$$

where $\left|\delta^{*}\right|$ is the length of $\delta^{*}$. Now

$$
R-\rho\left(I\left(z_{0}\right), C\right)=R^{2} /\left(\rho\left(z_{0}, C\right)+R\right)
$$

so

$$
1 / \rho\left(I\left(z_{0}\right), C\right)=\left(\rho\left(z_{0}, C\right)+R\right) / R \rho\left(z_{0}, C\right) \leqq \frac{\Delta / R+1}{\rho\left(z_{0}, C\right)},
$$

where $\Delta$ is the diameter of $D$.

Now look at $\rho\left(z_{0}, C\right)$ :

$$
\rho\left(z_{0}, C\right) \geqq \rho\left(z_{0}, \gamma\right)-2\left(R-\left(R^{2}-s^{2} / 4\right)^{1 / 2}\right)
$$

where $s=|\gamma(\alpha+\delta)-\gamma(\alpha)| \leqq \delta$. But $\delta$ was sufficiently small that

$$
2\left(R-\left(R^{2}-\delta^{2} / 4\right)^{1 / 2}\right)<\rho\left(z_{0}, \gamma\right) / 2,
$$

and since $s \leqq \delta$,

$$
2\left(R-\left(R^{2}-s^{2} / 4\right)^{1 / 2}\right) \leqq 2\left(R-\left(R^{2}-\delta^{2} / 4\right)^{1 / 2}\right) .
$$

Hence

$$
\rho\left(z_{0}, C\right)>\rho\left(z_{0}, \gamma\right) / 2, \text { also }\left|\delta^{*}\right| \leqq \frac{\pi}{2} s \leqq \frac{\pi}{2} \delta
$$

and it follows that

$$
W\left(z_{\tau} \in \gamma([a, a+\delta])\right) \leqq \frac{2}{2 \pi} \frac{\pi}{2} \delta \cdot 2(\Delta / R+1) / \rho\left(z_{0}, \gamma\right)=B \delta / \rho\left(z_{0}, \gamma\right) .
$$

4. Some inequalities. We shall need the following.

LEMMA.

$$
\begin{aligned}
W(\tau>t) & \leqq \frac{4}{\pi} \exp \left(-\pi^{2} t / 8 \Delta^{2}\right) \\
P^{n}(\tau>t) & \leqq \frac{4}{\pi} \exp \left(-\pi^{2} t / 8 \Delta^{2}\right)+A M(n t)^{-1 / 8}(\log n t)^{1 / 2},
\end{aligned}
$$


where $\Delta$ is the diameter of $D$ and $A$ is an absolute constant.

Proof.

$$
\begin{aligned}
W(\tau>t) & \leqq \operatorname{Pr}\left(\max _{0 \leqq s \leqq t}\left|z_{s}-z_{0}\right|<\Delta\right) \\
& \leqq \operatorname{Pr}\left(\max _{0 \leqq s \leqq 1}\left|\operatorname{Re}\left(z_{s}-z_{0}\right)\right|<\Delta / \sqrt{t}\right)=T(\Delta / \sqrt{t}) \\
& \leqq \frac{4}{\pi} \exp \left(-\pi^{2} t / 8 \Delta^{2}\right),
\end{aligned}
$$

where $T(\lambda)=\operatorname{Pr}\left(\max _{0 \leqq s \leqq 1}\left|x_{s}\right|<\lambda\right)$. The last inequality comes from the fact that the infinite series expansion for $T(\lambda)$ [11] is alternating, with decreasing terms.

$$
\begin{aligned}
P^{n}(\tau>t) & \leqq \operatorname{Pr} \max _{k \leqq n t}\left(\left|\zeta_{k}-z_{0}\right|<\Delta\right) \\
& \leqq \operatorname{Pr}\left(\max _{k \leqq n t} \mid \operatorname{Re}\left(\zeta_{k}-z_{0}\right) / \sqrt{t \mid}<\Delta / \sqrt{t}\right) .
\end{aligned}
$$

Now the theorem of Rosencrantz [10] applies [11] and we have

$$
\begin{aligned}
& \operatorname{Pr}\left(\max _{k \leqq n t}\left|\operatorname{Re}\left(\zeta_{k}-z_{0}\right) / \sqrt{t}\right|<\Delta / \sqrt{t}\right) \\
\leqq & A \cdot M(\log n t)^{1 / 2}(n t)^{-1 / 8}+T(\Delta / \sqrt{t})
\end{aligned}
$$

where $A$ is an absolute constant. But we saw above that

$$
T(\Delta / \sqrt{t}) \leqq \frac{4}{\pi} \exp \left(-\pi^{2} t / 8 \Delta^{2}\right)
$$

so

$$
P^{n}(\tau>t) \leqq \frac{4}{\pi} \exp \left(-\pi^{2} t / 8 \Delta^{2}\right)+A M(n t)^{-1 / 8}(\log n t)^{1 / 2}
$$

Now we need more notation. Let $K_{\lambda}=\gamma([0, \lambda])$, let $\left(z_{\tau} \in K_{\lambda}\right)^{\varepsilon, \tau} \subset$ $C([0, \infty), \not{C})$ be defined by $y \in\left(z_{\tau} \in K_{\lambda}\right)^{\varepsilon, \tau}$ iff $\exists z$ such that $z_{\tau} \in K_{\lambda}$ and (for $\tau=\tau(z)$ ) $\sup _{0 \leqq s \leq r}\left|y_{s}-z_{s}\right|<\varepsilon$. Let $\delta=\sqrt{\varepsilon}$, and let $K_{\lambda}^{\delta}=$ $\gamma([0, \lambda+\delta]) \cup \gamma(l-\delta, l])$, where $l$ is the length of $\gamma$.

\section{Proposition 3.}

$$
W\left(\left(z_{\tau} \in K_{\lambda}\right)^{\varepsilon, \tau} \cap\left(z_{\tau} \notin K_{\lambda}^{\delta}\right)\right) \leqq G \sqrt{\varepsilon}
$$

where $G$ is a constant depending only on $\gamma$.

Proof. Let $\tau(\partial \varepsilon)=\inf \left\{t: \rho\left(z_{t}, K_{\lambda}\right)<\varepsilon\right\}$ where $\rho\left(z_{t}, K_{\lambda}\right)$ is the distance from $z_{t}$ to $K_{\lambda}$. Then 


$$
\begin{aligned}
& W\left(\left(z_{\tau} \in K_{\lambda}\right)^{\varepsilon, \tau} \cap\left(z_{\tau} \notin K_{\lambda}^{\delta}\right)\right) \\
& \leqq W\left(\tau(\partial \varepsilon)<\tau, z_{\tau} \notin K_{\lambda}^{\delta}\right)+W\left(\tau(\partial \varepsilon)>\tau, z_{\tau} \notin K_{\lambda}^{\delta}, \tau(\partial \varepsilon)<\tau(s \varepsilon)\right) \\
&= E^{W}\left(\chi_{\tau(\delta \varepsilon)<\tau} P_{z_{\tau}(\partial \varepsilon)}\left(z_{\tau} \notin K_{\lambda}^{\gamma}\right)\right. \\
&\left.\quad+\chi_{\left[\tau(\partial \varepsilon)>\tau, z_{\tau} \notin K_{\lambda}\right]} P_{z_{\tau}}(\tau(\partial \varepsilon)<\tau(s \varepsilon))\right)
\end{aligned}
$$

by the strong Markov property [1, p. 268], where $\tau(s \varepsilon)=\inf \left\{t: \rho\left(z_{t}\right.\right.$, $D)>\varepsilon\}$. We estimate $P_{\left.z_{\tau(\partial \varepsilon}\right)}\left(z_{\tau} \notin K_{\lambda}^{\delta}\right)$ :

Let $\gamma(a)$ be a point in $K_{\lambda}$ of distance $\varepsilon$ from $z_{\tau\left(\partial_{\varepsilon}\right)}$, let $T$ be the tangent to $\gamma$ at $\gamma(a)$. Let $S_{i}(i=1,2)$ be lines perpendicular to $T$ through the points $\gamma(a-\delta)$ and $\gamma(a+\delta)$. The distance $d_{i}$ from $z_{\tau(\partial \varepsilon)}$ to each of the lines $S_{i}$ will be less than $\delta+\varepsilon$ (less than $\delta$ unless $\gamma(\alpha)$ is an endpoint of $K_{\lambda}$; let $d=\min \left(d_{1}, d_{2}\right)$. Let $T^{\prime}$ be parallel to $T$, at a distance $\varepsilon \cdot \sup \left|\gamma^{\prime \prime}\right|$ on the opposite side of $T$ from $z_{\tau(\partial \varepsilon)}$. I now claim $\gamma([a-\delta, a+\delta]) \cap T^{\prime}=\varnothing$ if $2 \delta<1 / \sup \left|\gamma^{\prime \prime}\right|$. Choose coordinates such that $\gamma(a)=0, \gamma^{\prime}(a)>0$. Then by Taylor's Theorem, for each $h$ there exists $\theta$ such that

$$
\begin{aligned}
\operatorname{Im} \gamma(a+h \delta) & =\operatorname{Im} \gamma(a)+\operatorname{Im} \gamma^{\prime}(a) \cdot h \delta+\operatorname{Im} \gamma^{\prime \prime}(a+\theta h \delta) \cdot h^{2} \delta^{2} / 2 \\
& =\operatorname{Im} \gamma^{\prime \prime}(a+\theta h \delta) \cdot h^{2} \delta^{2} / 2 .
\end{aligned}
$$

Hence for $|h| \leqq 1,|\operatorname{Im} \gamma(a+h \delta)| \leqq \sup \left|\delta^{\prime \prime}\right| \cdot \delta^{2} / 2<\varepsilon \cdot \sup \left|\gamma^{\prime \prime}\right|$ and $\gamma([a-\delta$, $a+\delta$ ]) does not meet $T^{\prime}$.

Let $\tau_{T^{\prime}}$ be the first time (after $\tau(\partial \varepsilon)$ ) that $z_{t}$ hits the line $T^{\prime}, \tau_{S}$ the first time (after $\tau(\partial \varepsilon))$ that $z_{t}$ hits $S_{1} \cup S_{2}$, and $c=\rho\left(z_{\varepsilon(\partial \varepsilon)}, T^{\prime}\right) \leqq$ $\varepsilon \cdot\left(\sup \left|\gamma^{\prime \prime}\right|+1\right)$. Note that $\tau_{T^{\prime}}$ and $\tau_{S}$ are independent, since the components of Brownian motion in the direction of $S_{i}$ and $T^{\prime}$ are independent. We can write

$$
\begin{aligned}
& P_{z_{\tau(\delta \delta)}}\left(z_{\tau} \notin K_{\lambda}^{\delta}\right)<P_{z_{\tau}(\delta \varepsilon)}\left(\tau_{T}^{\prime}>\tau_{S}\right)+O(\delta) \\
& \quad=\int_{0}^{\infty} P_{z_{\tau}(\partial \varepsilon)}\left(\tau_{S}<t\right) d_{t} P_{z_{\tau}(\partial \varepsilon)}\left(\tau_{T^{\prime}} \leqq t\right)+O(\delta) .
\end{aligned}
$$

Now

$$
\begin{aligned}
P_{z_{\tau}(\partial \varepsilon)}\left(\tau_{T^{\prime}} \leqq t\right) & =P\left(\sup _{0 \leqq s \leqq t} x_{s} \geqq c\right)=P\left(\sup _{0 \leqq s \leqq 1} x_{s} \geqq c / \sqrt{t}\right) \\
& =\sqrt{2 / \pi} \int_{c / \sqrt{t}}^{\infty} e^{-u^{2} / 2} d u
\end{aligned}
$$

(cf. e.g., [1, p. 287] and [8, p. 227]).

Hence

$$
\begin{aligned}
P_{z_{\tau}(\delta \varepsilon)} & \left(\tau_{T^{\prime}}>\tau_{S}\right)=\int_{0}^{\infty} P_{z_{\tau}(\partial \varepsilon)}\left(\tau_{S}<t\right) \sqrt{2 / \pi} \frac{1}{2} c t^{-3 / 2} e^{-c^{2} / t} d t \\
& \leqq \int_{0}^{\infty} P\left(\sup _{0 \leqq s \leqq t}\left|x_{s}\right|>d\right)(c / \sqrt{2 \pi}) t^{-3 / 2} e^{-c^{2} / 2 t} d t \\
& \leqq 2 \int_{0}^{\infty} P\left(\sup _{0 \leqq s \leqq 1} x_{s}>d / \sqrt{t}\right)(c / \sqrt{2 \pi}) t^{-3 / 2} e^{-c^{2} / 2 t} d t
\end{aligned}
$$


which by a straightforward computation, is bounded by $2\left(d^{2} / c^{2}+1\right)^{-1 / 2}$.

But I claim $d \sim \delta$ : choose coordinate such that $\gamma(a)=0, \gamma^{\prime}(a)=$ 1. Using Taylor's Theorem we get $\delta \geqq d \geqq \delta-\left(\sup \left|\gamma^{\prime \prime}\right| / 2\right) \delta^{2}-\varepsilon$, so $d \sim \delta$. And $\delta=\sqrt{\varepsilon}, c \leqq \varepsilon\left(\sup \left|\gamma^{\prime \prime}\right|+1\right)$, so

$$
2\left(d^{2} / c^{2}+1\right)^{-1 / 2} \leqq G_{1} \varepsilon / \delta=G_{1} \sqrt{\varepsilon}
$$

for some constant $G_{1}$.

Now the same argument can be applied to estimate $P_{z_{\tau}}(\partial \varepsilon)<$ $\tau(s \varepsilon)$ ) (i.e., the probability that a Brownian path will move a distance $d \sim \delta=\sqrt{\varepsilon}$ in the direction tangent to the curve before it moves a distance $c=O(\varepsilon)$ in the direction normal to the curve).

Hence $P_{z_{\tau}}(\tau(\partial \varepsilon)<\tau(s \varepsilon)) \leqq G_{2} \sqrt{\varepsilon}$ for some constant $G_{2}$ and our proposition follows.

5. Proof of the theorem. We are now ready to prove our theorem.

$$
\begin{aligned}
& \left|E^{P^{n}}(\Phi)-E^{W}(\Phi)\right| \\
& \quad=\left|E^{P^{n}}\left(\Phi \chi_{\tau \leqq t}\right)-E^{W}\left(\Phi \chi_{\tau \leqq t}\right)+E^{P^{n}}\left(\Phi \chi_{\tau>t}\right)-E^{W}\left(\Phi \chi_{\tau>t}\right)\right| \\
& \quad \leqq E^{P^{n}}\left(\Phi \chi_{\tau \leqq t}\right)-E^{W}\left(\Phi \chi_{\tau \leqq t}\right)+\sup _{r}|\phi|\left(P^{n}(\tau>t)+W(\tau>t)\right) .
\end{aligned}
$$

Looking at the first term,

$$
\begin{aligned}
&\left|E^{P^{n}}\left(\Phi \chi_{\tau \leqq t}\right)-E^{W}\left(\Phi \chi_{\tau \leqq t}\right)\right| \\
&=\left|\int_{0}^{l} \phi(\gamma(\lambda))\left(P^{n}\left(z_{\tau} \in \gamma(d \lambda), \tau \leqq t\right)-W\left(z_{\tau} \in \gamma(d \lambda), \tau \leqq t\right)\right)\right| \\
& \leqq|\phi(\gamma(0))|(P(\tau>t)+W(\tau>t))+\int_{0}^{l} \mid P^{n}\left(z_{\tau} \in K_{\lambda}, \tau \leqq t\right) \\
&-W\left(z_{\tau} \in K_{\lambda}, \tau \leqq t\right)|\cdot| d \phi(\lambda) \mid .
\end{aligned}
$$

We estimate the integrand:

The event $\left(z_{\tau} \in K_{\lambda}, \tau \leqq t\right)$ is determined by the behavior of the path up to time $t$, so

$$
\begin{aligned}
P^{n}\left(z_{\tau} \in K_{\lambda}, \tau \leqq t\right) & -W\left(z_{\tau} \in K_{\lambda}, \tau \leqq t\right)=P_{t}^{n}\left(z_{\tau} \in K_{\lambda}, \tau \leqq t\right) \\
& -W_{t}\left(z_{\tau} \in K_{\lambda}, \tau \leqq t\right) .
\end{aligned}
$$

We can use the corollary of Proposition 1 to get

$$
\begin{aligned}
P_{t}^{n}\left(z_{\tau} \in K_{\lambda}, \tau \leqq t\right) \leqq & W_{t}\left(\left(z_{\tau} \in K_{\lambda}, \tau \leqq t\right)^{\varepsilon, t}\right)+\varepsilon \\
\leqq W_{t}\left(z_{\tau} \in K_{\lambda}, \tau \leqq t\right) & +W_{t}\left(\left(z_{\tau} \in K_{\lambda}, \tau \leqq t\right)^{\varepsilon, t}\right. \\
& \left.\quad-\left(z_{\tau} \in K_{\lambda}^{\delta}, \tau \leqq t\right)\right)+W_{t}\left(z_{\tau} \in K_{\lambda}^{\delta}-K_{\lambda}, \tau \leqq t\right)+\varepsilon,
\end{aligned}
$$

where $\varepsilon=\varepsilon(n, t)=C M^{1 / 4} n^{-1 / 8} t^{3 / 8}(\log n t)^{15 / 8}$.

Now $y \in\left(z_{\tau} \in K_{\lambda}, \tau \leqq t\right)^{\varepsilon, t}$ means $\exists z$ such that $\tau \leqq t, z_{\tau} \in K_{\lambda}$, 
$\sup _{0 \leqq s \leqq t}\left|y_{s}-z_{s}\right|<\varepsilon . \quad$ As this condition does not depend on $y_{s}$ for $s>t$,

$$
\begin{aligned}
& W_{t}\left(\left(z_{\tau} \in K_{\lambda}, \tau \leqq t\right)^{\varepsilon, t}-\left(z_{\tau} \in K_{\lambda}^{\delta}, \tau \leqq t\right)\right) \\
& \quad \leqq W\left(\left(z_{\tau} \in K_{\lambda}, \tau \leqq t\right)^{\varepsilon, \tau}-\left(z_{\tau} \in K_{\lambda}^{\delta}, \tau \leqq t\right)\right) \\
& \left.\quad \leqq W\left(\left(z_{\tau} \in K_{\lambda}\right)\right)^{\varepsilon, \tau}-\left(z_{\tau} \in K_{\lambda}^{\delta}\right)\right)+W(\tau>t) .
\end{aligned}
$$

Applying Propositions 2 and 3, we then have

$$
\begin{aligned}
P^{n}\left(z_{\tau} \in K_{\lambda}, \tau\right. & \leqq t)-W\left(z_{\tau} \in K_{\lambda}, \tau \leqq t\right) \\
& \leqq\left(G+2 B / \rho\left(z_{0}, \gamma\right)\right) \sqrt{\varepsilon}+W(\tau>t) .
\end{aligned}
$$

We apply the above argument to the complement $\gamma-K_{\lambda}$ of $K_{\lambda}$ in $\gamma$.

$$
\begin{gathered}
P^{n}\left(z_{\tau} \in \gamma-K_{\lambda}, \tau \leqq t\right)-W\left(z_{\tau} \in \gamma-K_{\lambda}, \tau \leqq t\right) \\
\leqq\left(G+2 B / \rho\left(z_{0}, \gamma\right)\right) \sqrt{\varepsilon}+W(\tau>t) .
\end{gathered}
$$

It follows that

$$
\begin{aligned}
\mid P^{n}\left(z_{\tau} \in K_{\lambda}, \tau \leqq t\right) & -W\left(z_{\tau} \in K_{\lambda}, \tau \leqq t\right) \mid \leqq\left(G+2 B / \rho\left(z_{0}, \gamma\right)\right) \sqrt{\varepsilon} \\
& +W(\tau>t)+P^{n}(\tau>t) .
\end{aligned}
$$

We can now estimate the integral

$$
\begin{aligned}
& \int_{0}^{l}\left|P^{n}\left(z_{\tau} \in K_{\lambda}, \tau \leqq t\right)-W\left(z_{\tau} \in K_{\lambda}, \tau \leqq t\right)\right| \cdot|d \phi(\lambda)| \\
& \quad \leqq\left(\left(G+2 B / \rho\left(z_{0}, \gamma\right)\right) \sqrt{\varepsilon}+W(\tau>t)+P^{n}(\tau>t)\right) V(\phi)
\end{aligned}
$$

where $V(\phi)$ is the total variation of $\phi$ on $\gamma$.

Combining the results of (5.1), (5.2), and (5.3), (we have)

$$
\begin{aligned}
\mid E^{P^{n}}(\Phi) & -E^{W}(\Phi) \mid \leqq \phi(\gamma(0))\left(P^{n}(\tau>t)+W(\tau>t)\right. \\
& \left.+V(\phi)\left(G+2 B / \rho\left(z_{0}, \gamma\right)\right) \sqrt{\varepsilon}+W(\tau>t)+P^{n}(\tau>t)\right) \\
& +\sup _{\gamma}|\phi|\left(P^{n}(\tau>t)+W(\tau>t)\right) \\
\leqq & V(\phi)\left(G+2 \beta / \rho\left(z_{0}, \gamma\right)\right) \sqrt{\varepsilon}+\left(V(\phi)+2 \sup _{\gamma}|\phi|\right) \cdot\left(P^{n}(\tau>t)\right. \\
& +W(\tau>t)) .
\end{aligned}
$$

This estimate is minimized by choosing $t$ so as to balance the factors $\sqrt{\varepsilon}$ and $\left(P^{n}(\tau>t)+W(\tau>t)\right)$. So setting

$$
t=\min \left\{s: s \geqq \frac{1}{2}(\Delta / \pi)^{2} \log n, \text { sn an integer }\right\} \text {, }
$$

we get

$$
\begin{aligned}
& P^{n}(\tau>t)+W(\tau>t) \\
\leqq & \frac{8}{\pi} n^{-1 / 16}+A \cdot M n^{-1 / 8}\left(\frac{1}{2}(\Delta / \pi)^{2} \log n\right)^{1 / 8}(\log n t)^{1 / 2} \\
\leqq & A_{1} M n^{-1 / 16}(\log n)^{5 / 8}
\end{aligned}
$$


and

$$
\begin{aligned}
\sqrt{\varepsilon} & =\sqrt{C} M^{1 / 8} n^{-1 / 16} t^{3 / 16}(\log n t)^{15 / 16} \\
& \leqq \sqrt{C} M^{1 / 8} n^{-1 / 16} A_{2}(\log n)^{9 / 8}
\end{aligned}
$$

where $A_{1}, A_{2}$ are absolute constants. Hence

$$
\begin{aligned}
\left|E^{P^{n}}(\Phi)-E^{W}(\Phi)\right| & \leqq V(\phi) M \frac{G \Delta+2 B}{\rho\left(z_{0}, \gamma\right)} \sqrt{C} A_{2} n^{-1 / 16}(\log n)^{9 / 8} \\
& +\left(V(\phi)+2 \sup _{\gamma}|\phi|\right) A_{1} M n^{-1 / 16}(\log n)^{5 / 8} \\
\leqq & \left(3 V(\phi)+2 \inf _{\gamma}|\phi|\right) M\left(\frac{G \Delta+2 B}{\rho\left(z_{0}, \gamma\right)} \sqrt{C} A_{2}+A_{1}\right) n^{-1 / 16}(\log n)^{9 / 8}
\end{aligned}
$$

But integration is linear, so we may assume $\phi(p)=0$ for some $p$ in $\gamma$, as we are taking the difference of expectations.

Letting $C^{*}=3\left((G \Delta+2 B) \sqrt{C} A_{2}+\Delta A_{1}\right)$ we have

$$
\left|E^{F^{n}}(\Phi)-E^{W}(\Phi)\right| \leqq \frac{C^{*} V(\phi) M}{\rho\left(z_{0}, \gamma\right)} n^{-1 / 16}(\log n)^{9 / 8}
$$

CoRollary. If $O$ is any subset of $\gamma$ consisting of a finite number $k$ of intervals, then

$$
\left|P_{z_{0}}^{n}\left(z_{\tau} \in O\right)-W_{z_{0}}\left(z_{\tau} \in O\right)\right| \leqq \frac{2 k C^{*} M}{\rho\left(z_{0}, \gamma\right)} n^{-1 / 16}(\log n)^{9 / 8}
$$

\section{REFERENCES}

1. L. Breiman, Probability, Addison-Wesley, Reading, Massachusetts, 1968.

2. K. G. Esseen, Fourier analysis of distribution functions ... . Acta Math., 77 (1945), $1-125$.

3. W. Feller, An introduction to Probability Theory and its Applications, Volume II, Wiley, New York, 1966.

4. E. Hille, Analytic Function Theory, Volume I, Ginn, Boston, 1962.

5. K. Ito and H. P. McKean, Jr., Diffusion Processes and their Sample Paths, Academic Press, New York, 1965.

6. A. Kolmogorov, Über des Gesetz des iterierten Logarithmus, Math. Ann., 101 (1929), 126.

7. P. Lévy, Processus Stochastiques et Mouvement Brownien, Gauthier-Villars, Paris, 1965.

8. K. Parthasaraty, Probability Measures on Metric Spaces, Academic Press, New York, 1967.

9. Y. Prokhorov, Convergence of Random Processes and limit Theorems in Probability Theory, Theor. Prob. Appl., 1 (1956), 157-214.

10. W. Rosenkrantz, On Rates of Convergence for the Invariance Principle, Trans. Amer. Math. Soc., 129 (1967), 542-552.

11. S. Sawyer, A uniform rate of convergence for the maximum absolute value of partial sums in probability, Comm. Pure Appl. Math., 20 (1967), 647-658.

Received July 15, 1969 and in revised form May 15, 1972. This paper is a revision of a master's thesis written under the helpful guidance of Stanley A. Sawyer, while the author held a teaching assistantship at Brown University. 



\section{PACIFIC JOURNAL OF MATHEMATICS}

\section{EDITORS}

\section{H. SAMELSON}

Stanford University

Stanford, California 94305

\section{R. HOBBY}

University of Washington

Seattle, Washington 98105

\section{J. DugundjI}

Department of Mathematics University of Southern California Los Angeles, California 90007

RICHARD ARENS

University of California

Los Angeles, California 90024

\section{ASSOCIATE EDITORS}
E. F. BECKENBACH
B. H. NeumanN
F. WOLF
K. YOSHIDA

\section{SUPPORTING INSTITUTIONS}

\author{
UNIVERSITY OF BRITISH COLUMBIA \\ CALIFORNIA INSTITUTE OF TECHNOLOGY \\ UNIVERSITY OF CALIFORNIA \\ MONTANA STATE UNIVERSITY \\ UNIVERSITY OF NEVADA \\ NEW MEXICO STATE UNIVERSITY \\ OREGON STATE UNIVERSITY \\ UNIVERSITY OF OREGON \\ OSAKA UNIVERSITY
}

\author{
UNIVERSITY OF SOUTHERN CALIFORNIA \\ STANFORD UNIVERSITY \\ UNIVERSITY OF TOKYO \\ UNIVERSITY OF UTAH \\ WASHINGTON STATE UNIVERSITY \\ UNIVERSITY OF WASHINGTON \\ AMERICAN MATHEMATICAL SOCIETY \\ NAVAL WEAPONS CENTER
}

The Supporting Institutions listed above contribute to the cost of publication of this Journal, but they are not owners or publishers and have no responsibility for its content or policies.

Mathematical papers intended for publication in the Pacific Journal of Mathematics should be in typed form or offset-reproduced, (not dittoed), double spaced with large margins. Underline Greek letters in red, German in green, and script in blue. The first paragraph or two must be capable of being used separately as a synopsis of the entire paper. The editorial "we" must not be used in the synopsis, and items of the bibliography should not be cited there unless absolutely necessary, in which case they must be identified by author and Journal, rather than by item number. Manuscripts, in duplicate if possible, may be sent to any one of the four editors. Please classify according to the scheme of Math. Rev. Index to Vol. 39. All other communications to the editors should be addressed to the managing editor, Richard Arens, University of California, Los Angeles, California, 90024.

50 reprints are provided free for each article; additional copies may be obtained at cost in multiples of 50 .

The Pacific Journal of Mathematics is published monthly. Effective with Volume 16 the price per volume (3 numbers) is $\$ 8.00$; single issues, $\$ 3.00$. Special price for current issues to individual faculty members of supporting institutions and to individual members of the American Mathematical Society: $\$ 4.00$ per volume; single issues $\$ 1.50$. Back numbers are available.

Subscriptions, orders for back numbers, and changes of address should be sent to Pacific Journal of Mathematics, 103 Highland Boulevard, Berkeley, California, 94708.

PUBLISHED BY PACIFIC JOURNAL OF MATHEMATICS, A NON-PROFIT CORPORATION

Printed at Kokusai Bunken Insatsusha (International Academic Printing Co., Ltd.), 270, 3-chome Totsuka-cho, Shinjuku-ku, Tokyo 160, Japan. 


\section{Pacific Journal of Mathematics}

\section{Vol. 42, No. $3 \quad$ March, 1972}

Catherine Bandle, Extensions of an inequality by Pólya and Schiffer for vibrating membranes ................................ 543

S. J. Bernau, Topologies on structure spaces of lattice groups.......... 557

Woodrow Wilson Bledsoe and Charles Edward Wilks, On Borel product measures .......................................

Eggert Briem and Murali Rao, Normpreserving extensions in subspaces of

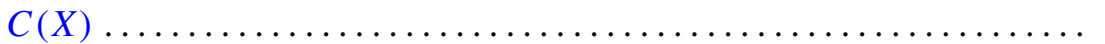

Alan Seymour Cover, Generalized continuation.................. 589

Larry Jean Cummings, Transformations of symmetric tensors .......... 603

Peter Michael Curran, Cohomology of finitely presented groups .......... 615

James B. Derr and N. P. Mukherjee, Generalized quasicenter and

hyperquasicenter of a finite group ...................... 621

Erik Maurice Ellentuck, Universal cosimple isols .................. 629

Benny Dan Evans, Boundary respecting maps of 3-mainfolds .......... 639

David F. Fraser, A probabilistic method for the rate of convergence to the

Dirichlet problem .................................. 657

Raymond Taylor Hoobler, Cohomology in the finite topology and Brauer

groups ..................................... 667

Louis Roberts Hunt, Locally holomorphic sets and the Levi form ........ 681

B. T. Y. Kwee, On absolute de la Vallée Poussin summability............ 689

Gérard Lallement, On nilpotency and residual finiteness in semigroups .... 693

George Edward Lang, Evaluation subgroups of factor spaces........... 701

Andy R. Magid, A separably closed ring with nonzero torsion pic ....... 711

Billy E. Rhoades, Commutants of some Hausdorff matrices ............. 715

Maxwell Alexander Rosenlicht, Canonical forms for local derivations . . . . 721

Cedric Felix Schubert, On a conjecture of L. B. Page ................ 733

Reinhard Schultz, Composition constructions on diffeomorphisms of $S^{p} \times S^{q}$

J. P. Singhal and H. M. (Hari Mohan) Srivastava, A class of bilateral generating functions for certain classical polynomials ....

Richard Alan Slocum, Using brick partitionings to establish conditions which insure that a Peano continuum is a 2-cell, a 2-sphere or an annulus...

James F. Smith, The p-classes of an $H^{*}$-algebra ...

Jack Williamson, Meromorphic functions with negative zeros and positive

poles and a theorem of Teichmuller ................. 